\title{
QCD Thermodynamics
}

\section{Zoltan Fodor}

John von Neumann Institute for Computing (NIC), DESY, D-15738, Zeuthen / FZJ, D-52425,

Juelich, Germany

Department of Physics, University of Wuppertal, Gauss Strasse 20, D-42119, Wuppertal, Germany

Institute for Theoretical Physics, Eotvos University, Pazmany 1, H-1117 Budapest, Hungary

Email: fodorabodri.elte.hu

Recent results on QCD thermodynamics are presented. The nature of the T $>0$ transition is determined, which turns out to be an analytic cross-over. The absolute scale for this transition is calculated. The temperature dependent static potential is given. The results were obtained by using a Symanzik improved gauge and stout-link improved fermionic action. In order to approach the continuum limit four different sets of lattice spacings were used with temporal extensions $N_{t}=4,6,8$ and 10 (they correspond to lattice spacings $a \sim 0.3,0.2,0.15$ and $0.12 \mathrm{fm}$ ). A new technique is presented, which -in contrast to earlier methods- enables one to determine the equation of state at very large temperatures.

The XXV International Symposium on Lattice Field Theory

July 30 - August 42007

Regensburg, Germany 


\section{Introduction}

The QCD transition at non-vanishing temperatures $(T)$ plays an important role in the physics of the early Universe and of heavy ion collisions (most recently at RHIC at BNL; LHC at CERN and FAIR at GSI will be the next generation of machines). The main goal of the present summary is to present results of the Budapest-Wuppertal group on the QCD transition at vanishing chemical potential $(\mu=0)$, which is of direct relevance for the early universe ( $\mu$ is negligible there) and for present and future heavy ion collisions (at RHIC $\mu \lesssim 40 \mathrm{MeV}$, which is far less than the typical hadronic scale). Since these results were obtained at four different sets of lattice spacings and a careful continuum extrapolation was performed, we consider them as full results. In addition, a new technique will be introduced, which closes the gap between lattice QCD and the perturbative approach for bulk thermodynamical quantities.

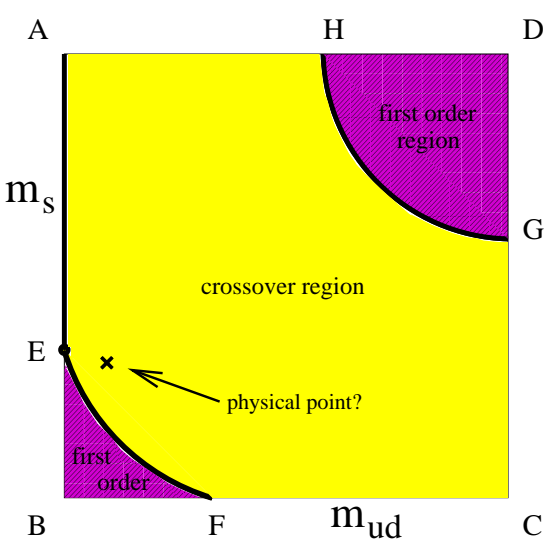

Figure 1: The phase diagram of QCD on the hypothetical light quark mass versus strange quark mass plane. Thick lines correspond to second order phase transitions, the purple regions represent first order phase transitions and the yellow region represents an analytic cross-over.
The standard picture for the QCD phase diagram on the light quark mass $\left(m_{u d}\right)$ versus strange quark mass $\left(m_{s}\right)$ plane is shown by Figure 1. It contains two regions at small and at large quark masses, for which the $T>0$ QCD transition is of first order. Between them one finds a cross-over region, for which the $T>0 \mathrm{QCD}$ transition is an analytic one. The first order transition regions and the cross-over region are separated by lines, which correspond to second order phase transitions.

When we analyze the nature and/or the absolute scale of the $T>0$ QCD transition for the physically relevant case two ingredients are quite important.

First of all, one should use physical quark masses. As Figure 1 shows the nature of the transition depends on the quark mass, thus for small or large quark masses it is a first order phase transition, whereas for intermediate quark masses it is an analytic cross over. Though in the chirally broken phase chiral perturbation theory provides a controlled technique to gain information for the quark mass dependence, it can not be applied for the $T>0$ QCD transition (which deals with the restoration of the chiral symmetry). In principle, the behaviour of different quantities in the critical region (in the vicinity of the second order phase transition line) might give some guidance. However, a priori it is not known how large this region is. Thus, the only consistent way to eliminate uncertainties related to non-physical quark masses is to use physical quark masses (which is, of course, quite CPU demanding).

Secondly, the nature of the $T>0$ QCD transition is known to suffer from discretization errors $[1,2,3]$. The three flavour theory with standard action on $N_{t}=4$ lattices predicts a critical pseudoscalar mass of about $300 \mathrm{MeV}$. This point separates the first order and cross-over regions of Figure 1. If we took another discretization, with another discretization error, e.g. the p4 action and $N_{t}=4$, the critical pseudoscalar mass turns out to be around $70 \mathrm{MeV}$ (similar effect is observed if one used stout smearing improvement and/or $\left.N_{t}=6\right)$. Since the physical pseudoscalar mass $(135 \mathrm{MeV})$ is just between these two values, the discretization errors in the first case would lead to a first order 

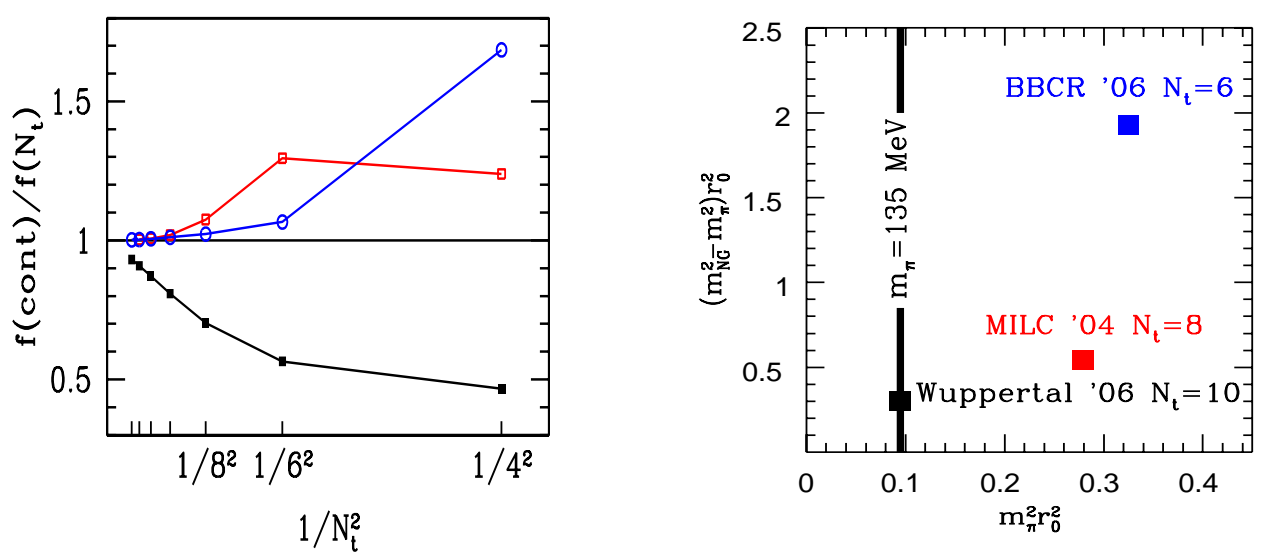

Figure 2: The ratio $f($ cont $) / f\left(N_{t}\right)$ as a function of $1 / N_{t}^{2}$ (left panel). $f($ cont $)$ is the continuum extrapolated free energy of the staggered fermionic gas in the non-interactive, infinitely high temperature limit. $f\left(N_{t}\right)$ is the value obtained on a lattice with $N_{t}$ temporal extension. The black line shows our choice (stout improvement, only next-neighbour terms in the action), whereas the red and blue lines represent the Naik and p4 actions, respectively. Masses and taste symmetry violation for different approaches in the literature (right panel). The smallest, physical quark mass and the smallest taste symmetry violation was reached by our works (black dot, [8,9]). Somewhat larger taste symmetry violation and about three times larger quark masses were reached by the MILC analysis on QCD thermodynamics (red dot, [10]). Even larger taste symmetry violation and about four times the physical quark masses are the characteristics of the BielefeldBrookhaven-Columbia-Riken result on $T_{c}$ (blue dot, [5]).

transition, whereas in the second case to a cross-over. The only way to resolve this inconclusive situation is to carry out a careful continuum limit analysis.

Since the nature of the transition influences the absolute scale $\left(T_{c}\right)$ of the transition -its value, mass dependence, uniqueness etc.- the above comments are valid for the determination of $T_{c}$, too.

Therefore, we have to answer the question: what happens for physical quark masses, in the continuum, at what $T_{c}$ ? In order to get a reliable answer we used physical quark masses on $N_{t}=4,6,8$ and 10 lattices, which correspond to approximately $0.3,0.2,0.15$ and $0.12 \mathrm{fm}$ lattice spacings, respectively.

In the presentation [4] of the results of the Bielefeld-Brookhaven-Columbia-Riken Collaboration published results [5] from $N_{t}=4$ and 6 were shown (and some unpublished figures for $N_{t}=8$, which were obtained within the HotQCD Collaboration). Since the CPU requirements for thermodynamics increase as $\approx N_{t}^{12}$ our $N_{t}=10$ simulations need about 50 times more CPU than $N_{t}=6$. Do we have 50 times more resources for QCD thermodynamics than our competitors? Of course not (it is almost the other way around). Instead, reaching $N_{t}=10$ is a fine balance. It is partly related to the choice of our action (which will be discussed in the next section), partly to the arrangements of the financial resources. For instance, as $N_{t}$ increases, one needs more and more statistics. Thus the thermalization can be done only once on a relatively expensive, scalable machine, such as BlueGene/L, whereas a large fraction of the non-vanishing $\mathrm{T}$ simulations can be done on more cost effective devices such as personal computer [6] graphics cards. A 2 years old model can accommodate $N_{t}=6$ lattices, on a one-and-a-half year old model you can put $N_{t}=8$ lattices and the one year 

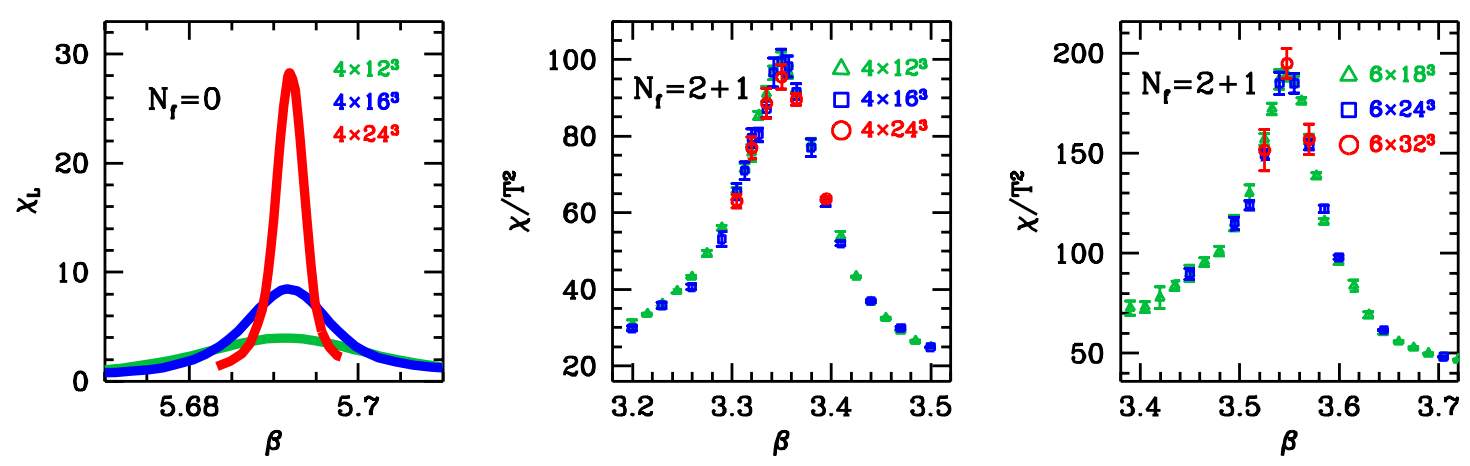

Figure 3: The volume dependence of the susceptibility peaks for pure SU(3) gauge theory (Polyakov-loop susceptibility, left panel) and for full QCD (chiral susceptibility on $N_{t}=4$ and 6 lattices, middle and right panels, respectively).

old model can work with quite large $N_{t}=10$ lattices. It costs a few hundred dollars and can provide upto 30-60 Gflops sustained QCD performance. They are not easy to code, adding two numbers needs 3 pages, but recently more efficient programming environments were introduced. Clearly, this type of hardware provides a very advantageous price-performance ratio for lattice QCD.

\section{The choice of the action}

The first step is to choose an action, which respects all the needs of a thermodynamic analysis. $\mathrm{T}=0$ simulations are needed to set the scale and for renormalization. $\mathrm{T}>0$ simulations are needed to map the behaviour of the system at non-vanishing temperatures. The action should maintain a balance between these two needs, leading to approximately the same uncertainties for both sectors (otherwise a large fraction of the CPU-power is used just for "over-killing" one of the two sectors). We used Symanzik improved gauge and stout improved 2+1 flavour staggered fermions [7] (due to the stout improvement we have only next-neighbour terms in the fermionic part of the action). The simulations were done along the line of constant physics. The parameters were tuned with a quite high precision, thus at all lattice spacings the $m_{K} / f_{k}$ and $m_{K} / m_{\pi}$ ratios were set to their experimental values with an accuracy better than $2 \%$.

The choice of the action has advantages and disadvantages. As we will see the advantages are probably more important than the disadvantages. The left panel of Figure 2 shows the continuum free energy devided by its value at a given $N_{t}$. A related plot is usually shown by the BielefeldBrookhaven-Columbia-Riken collaboration as a function of $N_{t}$. Since in staggered QCD most lattice corrections scale with $a^{2}$, which is proportional to $1 / N_{t}^{2}$, it is instructive to show this ratio as a function of $1 / N_{t}^{2}$, for our action, for Naik and for $\mathrm{p} 4$. Extrapolations from 4 and 6 always overshoot or undershoot. Clearly, the Naik and p4 actions reach the continuum value much faster than our choice, but the $a^{2}$ scaling appears quite early even for actions with next-neighbour interactions. Extrapolations from $N_{t}$ and $N_{t}+2$ with our action are approximately as good as $N_{t}$ with the p4 action (which was taylored to be optimal for this quantity, namely for the free energy at infinitely large temperatures). In practice, it means that our choice with $N_{t}=8,10$ gives approximately $2 \%$ error for the free energy. In a balanced analysis you do not need more, because the corresponding lattice spacings 0.15 and $0.12 \mathrm{fm}$ are most probably not fine enough to set the scale unambiguously 


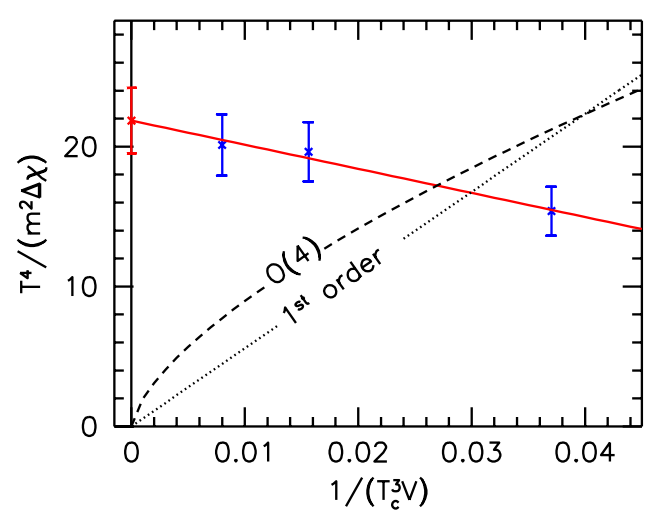

Figure 4: Continuum extrapolated susceptibilities $T^{4} /\left(\mathrm{m}^{2} \Delta \chi\right)$ as a function of $1 /\left(T_{c}^{3} V\right)$. For true phase transitions the infinite volume extrapolation should be consistent with zero, whereas for an analytic crossover the infinite volume extrapolation gives a non-vanishing value. The continuum-extrapolated susceptibilities show no phase-transition-like volume dependence, though the volume changes by a factor of five. The $\mathrm{V} \rightarrow \infty$ extrapolated value is 22(2) which is $11 \sigma$ away from zero. For illustration, we fit the expected asymptotic behaviour for first-order and $\mathrm{O}(4)$ (second order) phase transitions shown by dotted and dashed lines, which results in chance probabilities of $10^{-19}\left(7 \times 10^{-13}\right)$, respectively.

with the same accuracy. (E.g. the asqtad action at $N_{t} \approx 10$, which corresponds about a=0.12 fm lattice spacing, still has $\approx 10 \%$ scale difference between $r_{1} \& f_{K}$.) Since the $\mathrm{p} 4$ action is almost 20 times more expensive than the stout action, it is not worth to pay this price and improve one part of the calculation, which hinders you to reach a reasonable accuracy in another part of the calculation.

This is the balance one should remember in thermodynamics. Indeed, taste symmetry violation should be suppressed for many reasons (setting the scale at $T=0$, restoring chiral symmetry at $T>0$ etc.) As it was argued above it is more important to improve on this sector of the calculation than on the infinitely high temperature behaviour. The left panel of Figure 2 shows the splitting between the Goldstone and the first non-Goldstone pions for the Bielefeld-Brookhaven- ColumbiaRiken Collaboration (which is far beyond their kaon mass), for MILC and for us. Our small splitting is partly related to the stout improvement and partly to the cost issues, since smaller lattice spacings could be used, which resulted in smaller splitting. In addition, the cost issues allowed us to use in our finite $\mathrm{T}$ simulations physical quark masses instead of much larger masses.

\section{The nature of the QCD transition}

The next topic to be discussed is the nature of the QCD transition. Physical quark masses were used and a continuum extrapolation was carried out by using four different lattice spacings. The details of the calculations can be found in [8]. In order to determine the nature of the transition one should apply finite size scaling techniques for the chiral susceptibility $\chi=(T / V) \cdot\left(\partial^{2} \log Z / \partial m_{u d}^{2}\right)$. This quantity shows a pronounced peak as a function of the temperature. For a first order phase transition, such as in the pure gauge theory, the peak of the analogous Polyakov susceptibility gets more and more singular as we increase the volume (V). The width scales with $1 / \mathrm{V}$ the height scales with volume (see left panel of Figure 3). A second order transition shows a similar singular 
behaviour with critical indices. For an analytic transition (what we call a cross-over) the peak width and height saturates to a constant value. That is what we observe in full QCD on $N_{t}=4$ and 6 lattices (middle and right panels of Figure 3). We see an order of magnitude difference between the volumes, but a volume independent scaling. It is a clear indication for a cross-over. These results were obtained with physical quark masses for two sets of lattice spacings. Note, however, that for a final conclusion the important question remains: do we get the same volume independent scaling in the continuum; or we have the unlucky case what we had in the Introduction for 3 flavour QCD (namely the discretization errors changed the nature of the transition for the physical pseudoscalar mass case)?

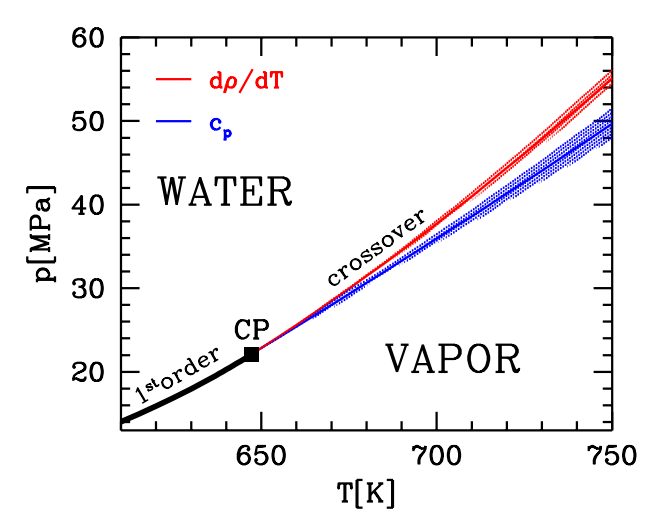

Figure 5: The water-vapor phase diagram.

We carried out a finite size scaling analyses with the continuum extrapolated height of the renormalized susceptibility. The renormalization of the chiral susceptibility can be done by taking the second derivative of the free energy density $(f)$ with respect to the renormalized mass $\left(m_{r}\right)$. We apply the usual definition: $f / T^{4}=-N_{t}^{4}$ $\cdot\left[\log Z\left(N_{s}, N_{t}\right) /\left(N_{t} N_{s}^{3}\right)-\log Z\left(N_{s 0}, N_{t 0}\right) /\left(N_{t 0} N_{s 0}^{3}\right)\right]$. This quantity has a correct continuum limit. The subtraction term is obtained at $T=0$, for which simulations are carried out on lattices with $N_{s 0}, N_{t 0}$ spatial and temporal extensions (otherwise at the same parameters of the action). The bare light quark mass $\left(m_{u d}\right)$ is related to $m_{r}$ by the mass renormalization constant $m_{r}=Z_{m} \cdot m_{u d}$. Note that $Z_{m}$ falls out of the combination $m_{r}^{2} \partial^{2} / \partial m_{r}^{2}=m_{u d}^{2} \partial^{2} / \partial m_{u d}^{2}$. Thus, $m_{u d}^{2}\left[\chi\left(N_{s}, N_{t}\right)-\chi\left(N_{s 0}, N_{t 0}\right)\right]$ also has a continuum limit (for its maximum values for different $N_{t}$, and in the continuum limit we use the shorthand notation $\left.m^{2} \Delta \chi\right)$.

In order to carry out the finite volume scaling in the continuum limit we took three different physical volumes (see Figure 4). The inverses of the volumes are shown in units of $T_{c}$. For these 3 physical volumes we calculated the dimensionless combination $T^{4} / \mathrm{m}^{2} \Delta \chi$ at 4 different lattice spacings: $0.3 \mathrm{fm}$ was always off, otherwise the continuum extrapolations could be carried out, which are shown on Figure 4. Our result is consistent with an approximately constant behaviour, despite the fact that we had a factor of 5 difference in the volume. The chance probabilities, that statistical fluctuations changed the dominant behaviour of the volume dependence are negligible. As a conclusion we can say that the staggered $\mathrm{QCD}$ transition at mu=0 is a cross-over.

\section{The transition temperature}

An analytic cross-over, like the QCD transition has no unique $T_{c}$. A particularly nice example for that is the water-vapor transition (c.f. Figure 5). Up to about $650 \mathrm{~K}$ the transition is a first order one, which ends at a second order critical point. For a first or second order phase transition the different observables (such as density or heat capacity) have their singularity (a jump or an infinitly high peak) at the same pressure. However, at even higher temperatures the transition is an analytic cross-over, for which the most singular points are different. The blue curve shows the 


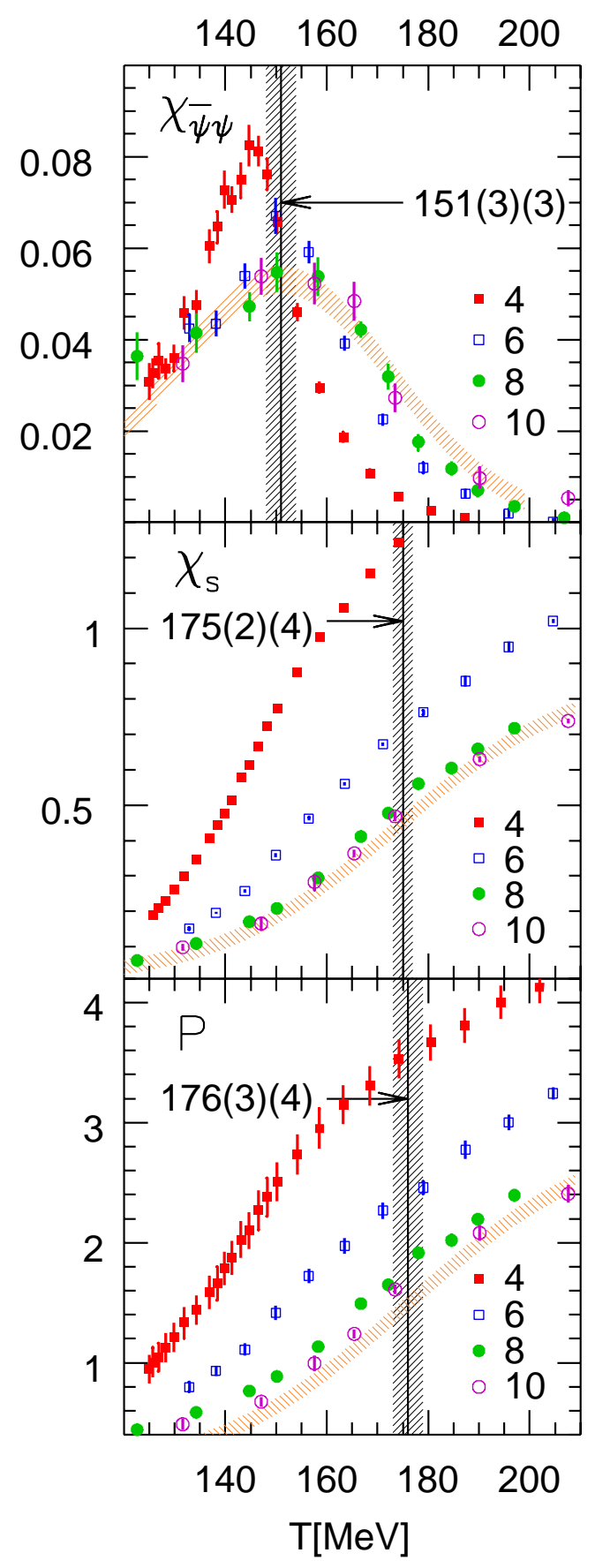

Figure 6: Temperature dependence of the renormalized chiral susceptibility $\left(m^{2} \Delta \chi_{\bar{\psi} \psi} / T^{4}\right)$, the strange quark number susceptibility $\left(\chi_{s} / T^{2}\right)$ and the renormalized Polyakov-loop $\left(P_{R}\right)$ in the transition region. The different symbols show the results for $N_{t}=4,6,8$ and 10 lattice spacings (filled and empty boxes for $N_{t}=4$ and 6, filled and open circles for $N_{t}=8$ and 10). The vertical bands indicate the corresponding transition temperatures and their uncertainties coming from the $\mathrm{T} \neq 0$ analyses. This error is given by the number in the first parenthesis, whereas the error of the overall scale determination is indicated by the number in the second parenthesis. The orange bands show our continuum limit estimates for the three renormalized quantities as a function of the temperature with their uncertainties. 
peak of the heat capacity and the red one the inflection point of the density. Clearly, these transition temperatures are different, which is a characteristic feature of an analytic transition (cross-over).

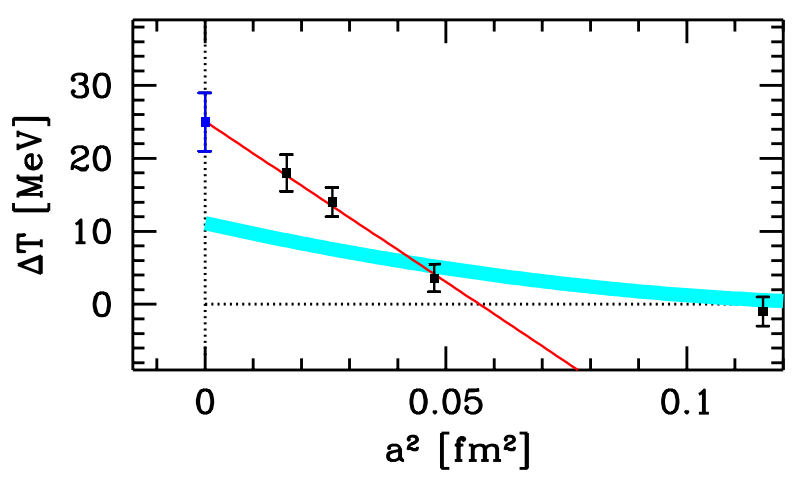

Figure 7: Difference between the the $T_{c}$ values obtained by the Polyakov loop and by the chiral condensate as a function of $a^{2}$.
In QCD we will study the chiral and the quark number susceptibilities and the Polyakov loop. Usually they give different $T_{c}$ values, but there is nothing wrong with it. As it was illustrated by the watervapor transition it is a physical ambiguity, related to the analytic behaviour of the transition. There is another, non-physical, ambiguity. If we used different observables (such as the string tension, $r_{0}$, the rho mass or the kaon decay constant), particularly at large lattice spacings we obtain different overall scales. They lead

to different $T_{c}$ values. This ambiguity disappeares in the continuum limit. According to our experiences, at finite lattice spacings, the best choice is the kaon decay constant $f_{k}$. It is known experimentally (in contrast to the string tension or $r_{0}$ ), thus no intermediate calculation with unknown systematics is involved. Furthermore it can be measured on the lattice quite precisely.

Figure 6 shows the results for the chiral susceptibility, for the quark number susceptibility and for the Polyakov loop. Red, blue, green, and purple indicate $N_{t}=4,6,8$ and 10 lattices. $N_{t}=4$ is always off, the rest scales nicely. The shaded regions indicate the continuum estimates. There is a surprising several sigma effect. The remnant of the chiral transition happens at a quite different temperature than that of the deconfining transition. It is quite a robust statement, since the Polyakov transition region is quite off the $\chi$-peak, and the $\chi$-peak is quite far from the inflection point of the Polyakov loop. This quite large differece is also related to the fact that the transition is fairly broad. The widths are around 30-40 MeV.

Due to the broadness of the transition the normalization prescription changes $T_{c}$, too. It is easy to imagine why, just multiply a Gaussian by $x^{2}$ and the peak is shifted. That means using $\chi / T^{2}$ gives about $10 \mathrm{MeV}$ higher $T_{c}$ than our definition, for which a $T^{4}$ normalization was applied. (Note, that for the unrenormalized $\chi$ a $T^{2}$ normalization is natural, whereas for the renormalized $\chi$ the natural normalization is done by $T^{4}$. This kind of naturalness manifests itself as possibly small errors of the observable.)

Figure 7 shows the difference between the $T_{c}$ values obtained by the Polyakov loop and by the chiral condensate as a function of the lattice spacing squared. The blue band indicates the difference for the chiral susceptibility peak position for the $T^{2}$ and $T^{4}$ normalization. Thus using the $T^{2}$ normalization no difference can be seen for $N_{t}=4$ and 6 , a slight difference is observed for $N_{t}=8$ and a reliable continuum extrapolation needs $N_{t}=6,8$ and 10 .

Our result on $T_{c}$ and that of the MILC Collaboration $\left(T_{c}=169(12)(4) \mathrm{MeV}[10]\right)$ are consistent within the (quite sizable) errorbars.

However, our result contradicts the recent Bielefeld-Brookhaven-Columbia-Riken result [5], which obtained 192(7)(4) MeV from both the chiral susceptibility and Polyakov loop. This value is about $40 \mathrm{MeV}$ larger than our result for the chiral susceptibility (for the Polyakov loop suscep- 


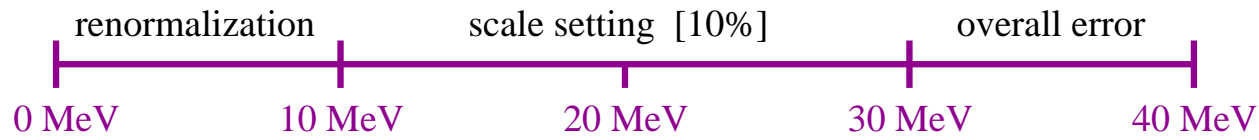

Figure 8: Possible contributions to the $40 \mathrm{MeV}$ difference between the results of Refs. [9] and [5].
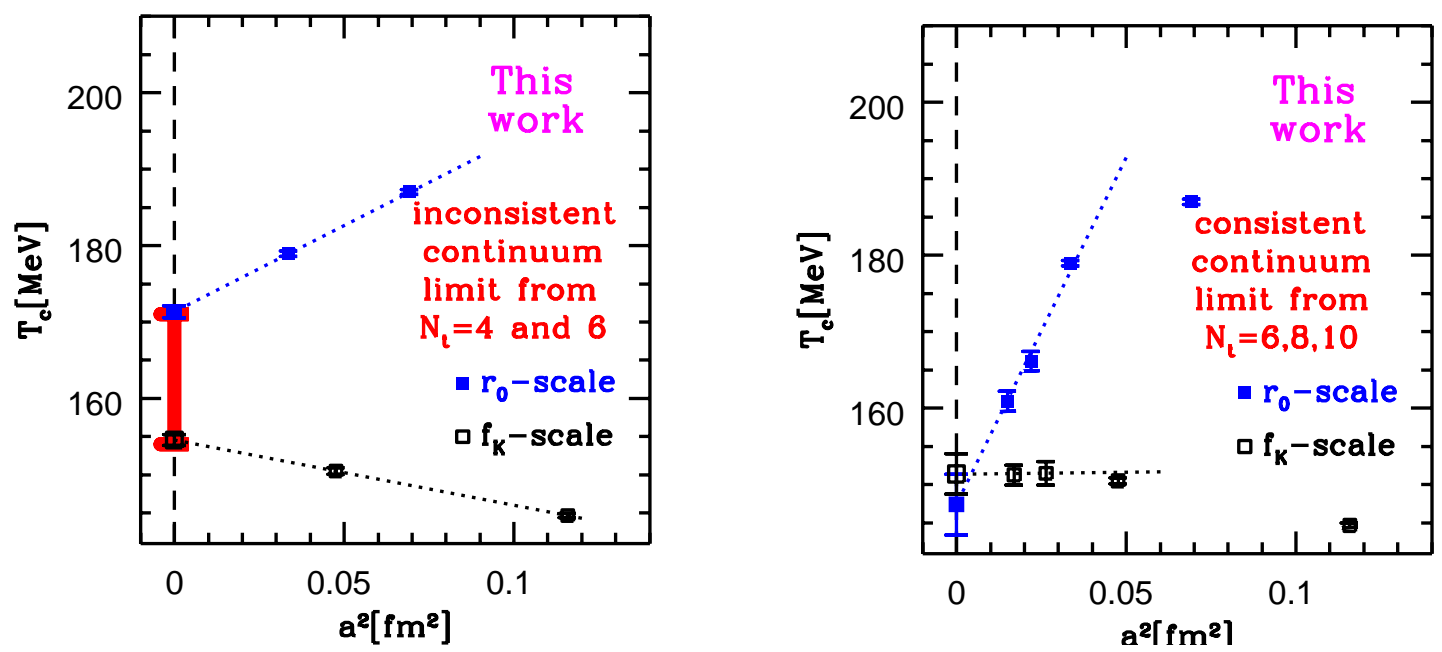

Figure 9: Continuum extrapolations based on $N_{t}=4$ and 6 (left panel: inconsistent continuum limit) and using $N_{t}=6,8$ and 10 (right panel: consistent continuum limit).

tibility the results agree within about $1.5 \sigma$ ). What are the differences between their analyses and ours, and how do they contribute to the $40 \mathrm{MeV}$ discrepancy? The most important contributions to the discrepancy are shown by Figure 8. The first difference is, that in [5] no renormalization was carried out, instead they used the unrenormalized quantity $\chi / T^{2}$. Due to the broadness of the distribution this observable leads to about $10 \mathrm{MeV}$ larger $T_{c}$ than our definition. The overall errors can be responsible for another $10 \mathrm{MeV}$. The origin of the remaining $20 \mathrm{MeV}$ is somewhat more complicated. One possible explanation can be summarized as follows. In Ref. [5] only $N_{t}=4$ and 6 were used, which correspond to lattice spacings $a=0.3$ and $0.2 \mathrm{fm}$, or $a^{-1}=700 \mathrm{MeV}$ and $1 \mathrm{GeV}$. These lattices are quite coarse and it seems to be obvious, that no unambiguous scale can be determined for these lattice spacings. The overall scale in Ref. [5] was set by $r_{0}$ and no cross-check was done by any other quantity independent of the static potential (e.g. $f_{k}$ ). This choice might lead to an ambiguity for the transition temperature, which is illustrated for our data on Figure 9. Using only $N_{t}=4$ and 6 the continuum extrapolated transition temperatures are quite different if one took $r_{0}$ or $f_{K}$ to determine the overall scale. This inconsistency indicates, that these lattice spacing are not yet in the scaling region (similar ambiguity is obtained by using the p4 action of [5]). Having $N_{t}=4,6,8$ and 10 results this ambiguity disappears (as usual $N_{t}=4$ is off), these lattice spacings are already in the scaling region (at least within our accuracy). This phenomenon is not surprising at all. As it was already mentioned e.g. the asqtad action at $N_{t} \approx 10$ (which corresponds to about $\mathrm{a}=0.12 \mathrm{fm}$ lattice spacing) has $\approx 10 \%$ scale difference predicted by $r_{1}$ or $f_{K}$. 
The ambiguity related to the inconsistent continuum limit is clearly unphysical, and it is resolved as we take smaller and smaller lattice spacings (c.f. Figure 9). The differences between the $T_{c}$ values for different observables are physical, it is a consequence of the cross-over nature of the QCD transition. There is another phenomenon, namely the volume dependence of $T_{c}$, which is also physical. Recently A. Bazavov and B. Berg studied [11] the volume dependence of $T_{c}$ in the pure SU(3) theory (for which the transition is a first order phase transition). They applied the usual periodic boundary condition (which approximates the thermodynamic limit in a very effective way) and also a disorder wall boundary condition. The difference between the $T_{c}$ values can be as large as $30 \mathrm{MeV}$ (c.f. Figure 10). Clearly, a detailed study of this phenomenon is needed for dynamical QCD.

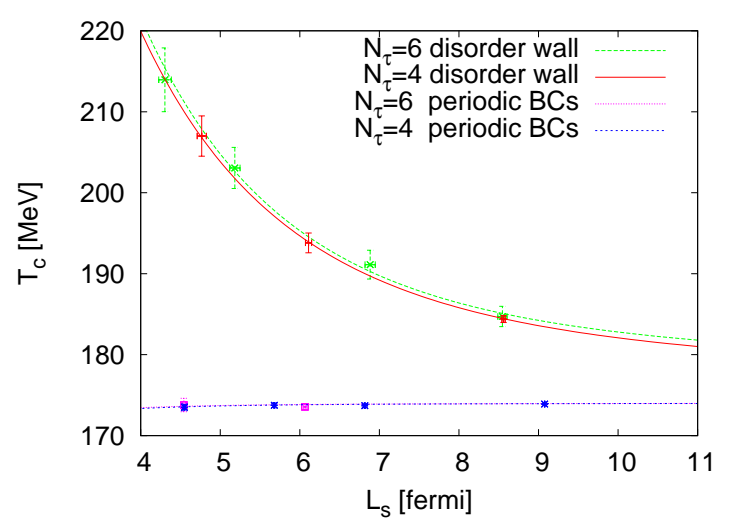

Figure 10: Transition temperature for the pure SU(3) theory as a function of the spatial size for $N_{t}=4$ and 6. The lower, almost horizontal lines represent the results with periodic boundary condition, whereas the upper curves show the results with disorder wall boundary condition (Figure from Ref. [11]).

\section{The renormalized static potential in the continuum limit}

Our aim is to compute the free energy of a static quark-antiquark pair. There are several measurements on this quantity in the literature (for recent publications cf. [12, 13, $14,15])$. Here we go beyond these computations (for details see [16]), we use physical quark masses and perform a careful continuum limit extrapolation with the necessary renormalization procedure.

The quark-antiquark free energy can be expressed as correlators of Polyakov loops:

$$
e^{-F_{\bar{q} q}(\mathbf{r}) / T} \sim \sum_{\mathbf{x}}\left\langle\operatorname{Tr} P(\mathbf{x}) \operatorname{Tr} P^{\dagger}(\mathbf{x}+\mathbf{r})\right\rangle,
$$

where $\mathbf{r}$ is a vector in the spatial direction, $T=1 /\left(N_{t} a\right)$ is the temperature and $\mathbf{x}$ runs over all the spatial lattice sites. $P$ is the Polyakov loop.

In full dynamical QCD at large distances it is favorable to generate a quark-antiquark pair from the vacuum, which then screens the color field between the two Polyakov loops [17]. From this point (the string breaking scale) the lowest energy level will be insensitive of the position of the heavy quarks, resulting in a constant free energy.

At finite temperature the above picture persists, but we can also have general expectations about the temperature dependence. Physically we expect that in a thermal vacuum it is easier to generate a quark-antiquark pair than at $T=0$, since there are thermally excited particles around which can scatter on the gluonic string between the static quark-antiquark pair. The gluonic string can more easily break into a dynamical quark-antiquark pair.

When we approach the continuum limit, the value of the un-renormalized free energy diverges. This is because in a single Polyakov loop the self-energy is divergent. We expect:

$$
\left.\langle\operatorname{Tr} P(x)\rangle\right|_{\mathrm{div}}=e^{-C(a) N_{t} a}=e^{-C(a) / T},
$$


where $C(a) \rightarrow \infty$ in the continuum limit. At finite $a$ the specific value of $C(a)$ has no physical meaning, since it depends on how we define the "divergent part" of the self-energy (renormalization scheme). In the literature there are several ways to fix this constant [14, 9]. A possible way of fixing $C(a)$ is to take a physical observable based on $F_{\bar{q} q}$, and require that it should be independent of $a$. In fact, the most useful quantity in our calculation was the constant value of the free energy after the string breaking/screening, at a fixed temperature (its value was set to $T_{0}=190 \mathrm{MeV}$ in the calculation). The value of $T_{0}=190 \mathrm{MeV}$ was motivated by the fact that it is already in the deconfined phase where the statistical errors of the free energy are much smaller than in the confined phase. For the calculation we used the gauge configurations of Ref [9]. Having determined the renormalized free energy for all lattice spacings, we could take the continuum limit by using the $N_{t}=4,6,8$ and 10 free energies, and extrapolating in $1 / N_{t}^{2} \sim a^{2} \rightarrow 0$. In the studied region $N_{t}=8$ and 10 results almost completely coincide. Therefore a safe extrapolation to $1 / N_{t}^{2}=0$ is possible. We estimate the systematic error of this extrapolation by comparing the results coming from $N_{t}=6,8,10$ extrapolation and $N_{t}=8,10$ extrapolation. The result for the renormalized free energy at different temperatures, including both the statistical and the systematic errors, can be seen on Figure 11.

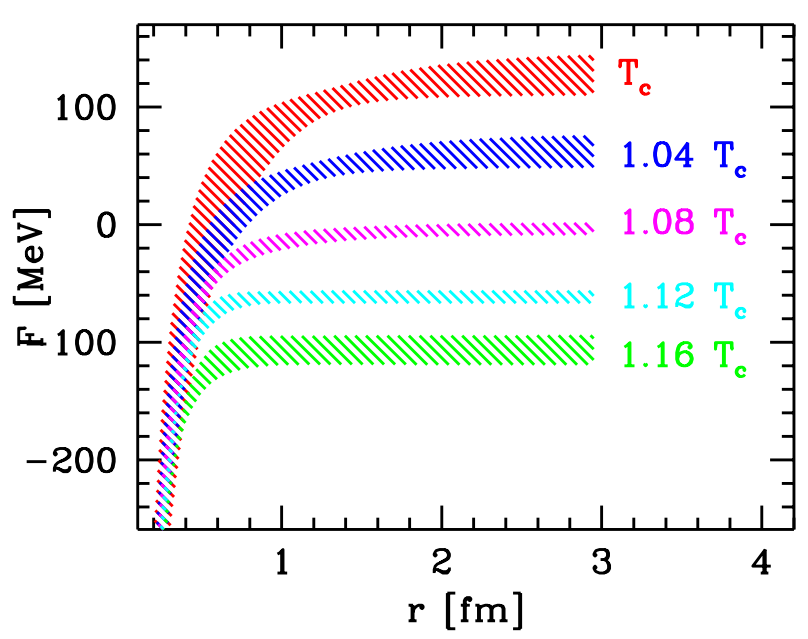

Figure 11: The renormalized free energies in the continuum limit.

\section{The equation of state at high temperatures}

While available lattice results for the equation of state (both for pure gauge theory and for full QCD) end at around $5 \cdot T_{c}$, standard perturbation theory converges only at extremely high temperatures (at $5 \cdot T_{c}$, the different perturbative orders can not even tell the sign of the deviation from the Stefan-Boltzmann limit). Until recently no link between the two most systematic methods of QCD, namely perturbation theory and lattice QCD, existed for bulk thermodynamical quantities.

It is easy to understand the difficulties for lattice calculations beyond these (e.g. $5 \cdot T_{c}$ ) temperatures. The correctly renormalized pressure is obtained by subtracting the vacuum term, thus $p_{\text {ren }}(T)=p(T)-p(T=0)$, where $p=T / V \cdot \log (Z)$ and $Z$ is the partition function. Since the partition function itself is difficult to determine, usually the integral method is used, which e.g. for the plaquette action is given by

$$
p_{\text {ren }}(T)=p(T)-p(T=0)=\int d \beta\left(\langle\mathrm{Pl}\rangle_{T}-\langle\mathrm{Pl}\rangle_{0}\right)
$$

As an example imagine a calculation at $20 \cdot T_{c}$ on $N_{t}=8$ lattices. A back of an envelope estimate can convince you that it corresponds to a lattice spacings of about $0.0075 \mathrm{fm}$ or lattice sizes of about 1000 in full QCD. This is clearly out of reach in the near future. 
In the perturbative approach the infrared properties of the non-abelian gauge theories at nonvanishing temperatures are responsible for the bad convergence.

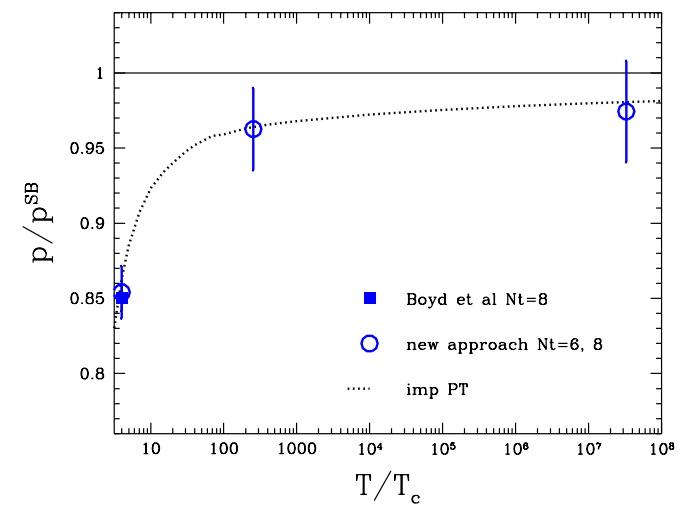

Figure 12: The pressure, normalized to its StefanBoltzmann value, as a function of the temperature obtained by our new technique. Results with small discretization errors $\left(N_{t}=8\right.$, blue circles), seem to fit to the improved perturbation theory prediction, and also reproduce results obtained by the standard method at lower temperatures. At the highest temperature, $3 \cdot 10^{7} \cdot T_{c}$, the pressure (within its statistical uncertainty) is consistent with the Stefan-Boltzmann limit.

In fact, we usually measure the dimensionless pressure, which can be obtained by including the $T^{4}$ factors. This leads to increasing powers of $1 / 2^{4}$ in the successive terms, thus only a few of them are needed. Applying this scheme, we may reach arbitrarily high temperatures using lattices with only $N_{t}$ and $2 \cdot N_{t}$ temporal extents. It is worth mentioning, that a similar formula can be constructed for the case of the normalized interaction measure $I \equiv(\varepsilon-3 \cdot p) / T^{4}$.

Note, that independently of the renormalization procedure, a problem emerges within the integral method framework. Since strictly speaking, the pressure is only exactly zero at $T=0$, in principle we would have to carry out the integration starting from zero temperature. Thus, there is an uncertainty of setting the lower point of the integral. This problem is solved by our direct method (c.f. [18]).

Let us consider the first bracketed term in $p_{\text {ren }}(T)$.

$$
[p(T)-p(T / 2)]=\frac{1}{N_{t} N_{s}^{3}} \log Z\left(N_{t}\right)-\frac{1}{2 N_{t} N_{s}^{3}} \log Z\left(2 N_{t}\right)=\frac{1}{2 N_{t} N_{s}^{3}} \log \left(\frac{Z\left(N_{t}\right)^{2}}{Z\left(2 N_{t}\right)}\right) .
$$

This can be determined by using $S_{1 b}$ (action with one boundary in the temporal direction), $S_{2 b}$ (action with two boundaries in the temporal direction) and an interpolating partition function $\bar{Z}(\alpha)=\int \mathscr{D} U \exp \left[-\left(\alpha \cdot S_{2 b}+(1-\alpha) \cdot S_{1 b}\right)\right]$ Using $\bar{Z}(\alpha)$, one obtains

$$
[p(T)-p(T / 2)] \sim \log \left(\frac{Z\left(N_{t}\right)^{2}}{Z\left(2 N_{t}\right)}\right)=\log \left(\frac{\bar{Z}(1)}{\bar{Z}(0)}\right)=\int_{0}^{1} d \alpha \frac{d \log \bar{Z}(\alpha)}{d \alpha}=\int_{0}^{1} d \alpha\left\langle S_{1 b}-S_{2 b}\right\rangle
$$


So we can calculate the pressure itself at any given temperature, without carrying out simulations at lower temperatures, and then performing an integral. (Note, however, that huge cancellations appear also within this framework).

The method discussed above gives us the possibility to measure the pressure at very high temperatures. This was carried out using lattices with temporal extension $N_{t}=4, N_{t}=6$ and $N_{t}=8$. The temperature interval ranged from $4 \cdot T_{c}$ upto $3 \cdot 10^{7} \cdot T_{c}$. Our results are shown on figure 12. A comparison can be done with the standard method, namely the result of [19] for smaller temperatures. Here we present the results which are closest to the continuum limit, thus $N_{t}=8$. These results nicely follow the perturbative predictions ( for a recent paper see [20] and references therein).

\section{Conclusion}

QCD thermodynamics results from the Budapest-Wuppertal group were summarized. The necessary balance between $T=0$ and $T>0$ simulations was discussed in detail. As a consequence, Symanzik improved gauge and stout-link improved staggered fermionic lattice action was used in the simulations with an exact simulation algorithm. Physical masses were taken both for the light quarks and for the strange quark. The parameters were tuned with a quite high precision, thus at all lattice spacings the $m_{K} / f_{k}$ and $m_{K} / m_{\pi}$ ratios were set to their experimental values with an accuracy better than $2 \%$. Four sets of lattice spacings on lattices with $N_{t}=4,6,8$ and 10 temporal extensions were used (they correspond to lattice spacing $a \sim 0.3,0.2,0.15$ and $0.12 \mathrm{fm}$ ) to carry out the continuum extrapolation. It turned out that only $N_{t}=6,8$ and 10 can be used for a controlled extrapolation, $N_{t}=4$ is out of the scaling region.

The nature of the $\mathrm{T}>0$ transition was determined. The renormalized chiral susceptibility was extrapolated to vanishing lattice spacing for three physical volumes, the smallest and largest of which differ by a factor of five. This ensures that a true transition should result in a dramatic increase of the susceptibilities. No such behaviour is observed: the finite-size scaling analysis showed that the finite-temperature QCD transition in the hot early Universe was not a real phase transition, but an analytic crossover (involving a rapid change, as opposed to a jump, as the temperature varied). As such, it will be difficult to find experimental evidence of this transition from astronomical observations. Since for present day heavy ion experiments the baryonic chemical potential is also very small, the above results apply for them, too.

The absolute scale for the T $>0$ transition was calculated. Since the QCD transition is a nonsingular cross-over there is no unique $T_{c}$. This well-known phenomenon was illustrated on the water-vapor phase diagram. Different observables led to different numerical $T_{c}$ values in the continuum and thermodynamic limit also in QCD. Three observables were used to determine the corresponding transition temperatures. The peak of the renormalized chiral susceptibility predicted $T_{c}=151(3)(3) \mathrm{MeV}$, whereas $T_{c}$ based on the strange quark number susceptibility resulted in 24(4) MeV larger value. Another quantity, which is related to the deconfining phase transition in the large quark mass limit is the Polyakov loop. Its behavior predicted a 25(4) MeV larger transition temperature, than that of the chiral susceptibility. Another consequence of the cross-over are the non-vanishing widths of the peaks even in the thermodynamic limit, which were also determined. 
For the chiral susceptibility, strange quark number susceptibility and Polyakov-loop we obtained widths of 28(5)(1) MeV, 42(4)(1) MeV and 38(5)(1) MeV, respectively.

The temperature dependent static potential was given. The same action and the same set of configurations were used as for the determination of $T_{c}$. Since results for different lattice spacings were available a careful renormalization program was carried out.

These features, numbers and functions are attempted to be the full result for the $T \neq 0$ transition, though other lattice fermion formulations - e.g. Wilson fermions (for ongoing projects see e.g. $[21,22]$ ) or chiral fermions (for an early dynamical overlap test see [23], for the domain wall approach a recent presentation can be found in Ref.[24]) — are needed to cross-check the findings with staggered fermions.

A new technique is presented, which -in contrast to earlier methods- enables one to determine the equation of state at very large temperatures. The method is based on the observation, that the divergences, which are removed by renormalization, are independent of the temperature. Thus the subtraction of the vacuum term, which is needed for renormalization, can be done at non-vanishing temperatures (actually even at very high temperatures). A direct method was also suggested, which does not need an integration over the temperature. Results for the pure SU(3) theory are presented upto $3 \cdot 10^{7} \cdot T_{c}$ temperatures.

\section{Acknowledgment}

Partial support of grants of DFG F0 502/1, EU I3HP, OTKA AT049652 and OTKA K68108 is acknowledged. The author thanks R. Hoffmann, S.D. Katz and K.K. Szabo for careful reading of the manuscript.

\section{References}

[1] F. Karsch et al., "Where is the chiral critical point in 3-flavor QCD?," Nucl. Phys. Proc. Suppl. 129 (2004) 614 [arXiv:hep-lat/0309116].

[2] O. Philipsen and P. De Forcrand, The chiral critical point of $N_{f}=3$ QCD: towards the continuum, PoS(LATTICE 2007)178

[3] G. Endrodi, Z. Fodor, S.D. Katz and K.K. Szabo, The nature of the finite temperature QCD transition as a function of the quark masses, PoS(LATTICE 2007)182

[4] F. Karsch, Recent lattice results on finite temperature and density QCD, PoS(LATTICE 2007)015

[5] M. Cheng et al., “The transition temperature in QCD,” Phys. Rev. D 74 (2006) 054507 [arXiv:hep-lat/0608013].

[6] G. I. Egri, Z. Fodor, C. Hoelbling, S. D. Katz, D. Nogradi and K. K. Szabo, "Lattice QCD as a video game," Comput. Phys. Commun. 177 (2007) 631 [arXiv:hep-lat/0611022].

[7] Y. Aoki, Z. Fodor, S. D. Katz and K. K. Szabo, “The equation of state in lattice QCD: With physical quark masses towards the continuum limit,” JHEP 0601 (2006) 089 [arXiv:hep-lat/0510084].

[8] Y. Aoki, G. Endrodi, Z. Fodor, S. D. Katz and K. K. Szabo, "The order of the quantum chromodynamics transition predicted by the standard model of particle physics," Nature 443 (2006) 675 [arXiv:hep-lat/0611014]. 
[9] Y. Aoki, Z. Fodor, S. D. Katz and K. K. Szabo, “The QCD transition temperature: Results with physical masses in the continuum limit," Phys. Lett. B 643 (2006) 46 [arXiv:hep-lat/0609068].

[10] C. Bernard et al. [MILC Collaboration], "QCD thermodynamics with three flavors of improved staggered quarks,” Phys. Rev. D 71 (2005) 034504 [arXiv:hep-lat/0405029].

[11] A. Bazavov and B. A. Berg, "Deconfining Phase Transition on Lattices with Boundaries at Low Temperature,” Phys. Rev. D 76 (2007) 014502 [arXiv:hep-lat/0701007].

[12] O. Kaczmarek, F. Karsch, P. Petreczky and F. Zantow, "Heavy quark anti-quark free energy and the renormalized Polyakov loop,” Phys. Lett. B 543 (2002) 41 [arXiv:hep-lat/0207002].

[13] V. Bornyakov et al., "Heavy quark potential in lattice QCD at finite temperature," arXiv:hep-lat/0301002.

[14] O. Kaczmarek, F. Karsch, F. Zantow and P. Petreczky, "Static quark anti-quark free energy and the running coupling at finite temperature,” Phys. Rev. D 70 (2004) 074505 [Erratum-ibid. D 72 (2005) 059903] [arXiv:hep-lat/0406036].

[15] Y. Maezawa, N. Ukita, S. Aoki, S. Ejiri, T. Hatsuda, N. Ishii and K. Kanaya [WHOT-QCD Collaboration], "Heavy-Quark Free Energy, Debye Mass, and Spatial String Tension at Finite Temperature in Two Flavor Lattice QCD with Wilson Quark Action,” Phys. Rev. D 75 (2007) 074501 [arXiv:hep-lat/0702004].

[16] Z. Fodor, A. Jakovac, S. D. Katz and K. K. Szabo, "Static quark free energies at finite temperature," arXiv:0710.4119 [hep-lat].

[17] G. S. Bali, H. Neff, T. Duessel, T. Lippert and K. Schilling [SESAM Collaboration], "Observation of string breaking in QCD,” Phys. Rev. D 71 (2005) 114513 [arXiv:hep-lat/0505012].

[18] G. Endrodi, Z. Fodor, S. D. Katz and K. K. Szabo, "The equation of state at high temperatures from lattice QCD," arXiv:0710.4197 [hep-lat].

[19] G. Boyd, et al., “Thermodynamics of SU(3) Lattice Gauge Theory,” Nucl. Phys. B469 (1996) 419-444 [arXiv:hep-lat/9602007].

[20] M. Laine and Y. Schroder, "Quark mass thresholds in QCD thermodynamics," Phys. Rev. D 73 (2006) 085009 [arXiv:hep-ph/0603048].

[21] Y. Maezawa et al., "Thermodynamics and heavy-quark free energies at finite temperature and density with two flavors of improved Wilson quarks," arXiv:0710.0945 [hep-lat].

[22] V. Weinberg, E. M. Ilgenfritz, K. Koller, Y. Koma, Y. Nakamura, G. Schierholz and T. Streuer [DIK Collaboration], "The chiral transition on a $24^{3} 10$ lattice with $N_{f}=2$ clover sea quarks studied by overlap valence quarks,” arXiv:0710.2565 [hep-lat].

[23] Z. Fodor, S. D. Katz and K. K. Szabo, "Dynamical overlap fermions, results with hybrid Monte-Carlo algorithm,” JHEP 0408, 003 (2004) [arXiv:hep-lat/0311010].

[24] P. Vranas, “The $N_{t}=8$ QCD thermal transition with DWF," PoS(LATTICE 2007)235. 